\title{
Solving the solar neutrino puzzle with KamLAND and solar data
}

\author{
André de Gouvêa \\ Theory Division, CERN, CH-1211 Geneva 23, Switzerland \\ Carlos Peña-Garay \\ Instituto de Física Corpuscular, Universitat de València - C.S.I.C. Edificio Instituto de Paterna, Apt. 22085, 46071 València, España
}

(Received 30 July 2001; published 9 November 2001)

\begin{abstract}
We study what will be learned about the solar neutrino puzzle and solar neutrino oscillations once the data from the KamLAND reactor neutrino experiment (soon to become available) are combined with those from the current solar neutrino experiments. We find that, in agreement with previous estimates, if the solution to the solar neutrino puzzle falls on the LMA region, KamLAND should be able to "pinpoint" the right solution with unprecedented accuracy after a few years of data taking. Furthermore, the light side $(\theta<\pi / 4)$ can be separated from the dark side $(\theta>\pi / 4)$ at the $95 \%$ confidence level (C.L.) for most of the LMA region allowed by the current data at the $99 \%$ C.L., while the addition of the KamLAND data need not improve our ability to limit a sterile component in "solar" oscillations. If KamLAND does not see an oscillation signal, the solar data would point to the LOW or VAC regions, while the SMA region would still lurk at the two sigma C.L., meaning we would probably have to wait for Borexino data in order to finally piece the solar neutrino puzzle.

DOI: 10.1103/PhysRevD.64.113011

PACS number(s): 14.60.Pq, 12.15.Ff, 96.60.Jw
\end{abstract}

\section{INTRODUCTION}

The $30+$ year old solar neutrino puzzle constitutes, together with the atmospheric neutrino puzzle, the only clean evidence for physics beyond the standard model. What started off as a discrepancy between the expected and experimentally measured solar neutrino flux at the chlorine experiment in the Homestake mine ( [1], and references therein) has now turned into, when the state-of-the-art SuperKamiokande [2] and SNO experiments [3] are combined, strong evidence that active neutrinos other than $\nu_{e}$ (i.e., $\nu_{\mu}$ and/or $\nu_{\tau}$ ) are part of the solar neutrino flux [3].

However, when the solar neutrino puzzle is taken as evidence for neutrino oscillations, many issues remain. First, the solar neutrino data is yet to provide unambiguous evidence for oscillations, and even the evidence for neutrino conversion requires the combination of two different experiments (this issue will soon be resolved after SNO measures neutral current events). Second, the current data is still unable to establish what is the generic value of the neutrino oscillation parameters [4-7]. Even if the recently disfavored small mixing angle (SMA) region is discarded, there are still a few disjoined values of $\Delta m^{2}$ which properly fit all the data. The (preferred) large mixing angle (LMA) region contains values of $10^{-5} \mathrm{eV}^{2} \lesssim \Delta m^{2} \lesssim 10^{-3} \mathrm{eV}^{2}$ and $0.2 \leq \tan ^{2} \theta \leq 3$, while the "low probability" (LOW), quasivacuum (QVO), and just-so (VAC) regions contain $10^{-11} \mathrm{eV}^{2} \lesssim \Delta m^{2}$ $\lesssim 10^{-6} \mathrm{eV}^{2}$ and $0.1 \lesssim \tan ^{2} \theta \lesssim 10$. Finally, it is still not clear whether the $\nu_{e}$ is oscillating into an active neutrino or a sterile neutrino. While the current data strongly disfavors pure $\nu_{e} \leftrightarrow \nu_{s}$ oscillations $\left[\nu_{s}\right.$ is a $S U(2) \times U(1)_{Y}$ singlet, sterile neutrino], the possibility that $\nu_{e}$ oscillates into a strongly mixed active $\oplus$ sterile state is quite robust $[8,9]$.

The KamLAND reactor neutrino experiment [10], which is to start taking data soon, is sensitive to the LMA region of the solar neutrino parameter space. After a few years of data taking, it is capable of either excluding the entire region or not only establishing $\nu_{e} \leftrightarrow \nu_{\text {other }}$ oscillations, but also of measuring the oscillation parameters $\left(\tan ^{2} \theta, \Delta m^{2}\right)$ with unprecedented precision [11-14].

Even in the case of a positive signal for oscillations at KamLAND, a few outstanding issues will remain. Because KamLAND is sensitive to $\bar{\nu}_{e}$ charged current events only, it is unable to tell whether the $\nu_{e}$ oscillates into an active or a sterile state. Furthermore, because it is a "short" baseline experiment, it is insensitive to matter effects, and therefore cannot separate the "light" from the "dark" side of the parameter space [15] (i.e., tell $\theta$ from $\pi / 2-\theta$ ).

In this paper, we try to determine what extra information will be gained when the KamLAND results are combined with the current solar neutrino data collected by Homestake, Sage, Gallex, GNO, Kamiokande-SuperKamiokande, and SNO $[1-3,16]$. In particular, we find that, for three years of KamLAND "data:" (i) for a significant portion of the LMA region, we should be able to separate the light from the dark side at more than $95 \%$ confidence level (C.L.), given that the KamLAND data is precise enough to rule out maximal mixing with the same precision; (ii) if KamLAND sees a signal, we can establish that the $\nu_{s}$ component of the "other" neutrino is smaller than $\lesssim 0.6$ at the $99 \%$ C.L. This bound, however, is sensitive to the values of $\Delta m^{2}$ and $\tan ^{2} \theta$ which are to be measured at KamLAND. If KamLAND does not see a signal, this upper bound is 0.78 at the $99 \%$ C.L. (iii) if KamLAND does not see a signal for oscillations, the solar data will point to the LOW, QVO, or VAC regions, while the SMA region should still be allowed at the (2-3) sigma level.

Our presentation is as follows. In the next section, we briefly review the KamLAND experiment, and discuss the details of our simulations of KamLAND data and data analysis. In Sec. III, we discuss the consequences of combining KamLAND and solar data if there is evidence for $\bar{\nu}_{e}$ disappearance at KamLAND, while in Sec. IV we discuss the status of the solar neutrino puzzle in the advent that Kam- 
LAND sees no suppression of the reactor $\bar{\nu}_{e}$ flux. In Sec. V we summarize our results and conclude.

\section{KamLAND EXPERIMENT AND “DATA" ANALYSIS}

The KamLAND detector, located inside a mine in Japan (in the site of the old Kamiokande experiment), consists of roughly 1 kton of liquid scintillator surrounded by photomultiplier tubes. It is sensitive to the $\bar{\nu}_{e}$ flux from some $10+$ reactors which are located "nearby." The distances from the different reactors to the experimental site vary from slightly more than $80 \mathrm{~km}$ to over $800 \mathrm{~km}$, while the majority (roughly 80\%) of the neutrinos travel from $140 \mathrm{~km}$ to 215 km (see, e.g., [10] for details). KamLAND "sees" the antineutrinos by detecting the total energy deposited by recoil positrons, which are produced via $\bar{\nu}_{e}+p \rightarrow e^{+}+n$. The total visible energy corresponds to $E_{e^{+}}+m_{e}$, where $E_{e^{+}}$is the kinetic energy of the positron and $m_{e}$ the electron mass. The positron energy, on the other hand, is related to the incoming antineutrino energy $E_{e^{+}}=E_{\nu}-1.293 \mathrm{MeV}$ up to corrections related to the recoil momentum of the daughter neutron (1.293 MeV is the neutron-proton mass difference). KamLAND is expected to measure the visible energy with a resolution which is expected to be better than $\sigma(E) / E$ $=10 \% / \sqrt{E}$, for $E$ in $\mathrm{MeV}[10,14]$.

In order to simulate events at KamLAND, we need to compute the expected energy spectrum for the incoming reactor antineutrinos for different values of the neutrino masssquared differences and mixing angles.

The antineutrino spectrum which is to be measured at KamLAND depends on the power output and fuel composition of each reactor (both change slightly as a function of time), and on the cross section for $\bar{\nu}_{e}+p \rightarrow e^{+}+n$. For the results presented here, we assume a constant chemical composition for the fuel of all reactors (explicitly, 53.8\% of ${ }^{235} \mathrm{U}$, $32.8 \%$ of ${ }^{239} \mathrm{Pu}, 7.8 \%$ of ${ }^{238} \mathrm{U}$, and $5.6 \%$ of ${ }^{241} \mathrm{Pu}$, see $[11,17])$. Effects due to the time variation of the fuel composition have been studied in [13], and are small (although they have to be taken into account in the "real" data analysis, of course).

The shape of energy spectrum of the incoming neutrinos can be derived from a phenomenological parametrization, obtained in [18],

$$
\frac{\mathrm{d} N_{\bar{\nu}_{e}}}{\mathrm{~d} E_{\nu}} \propto e^{a_{0}+a_{1} E_{\nu}+a_{2} E_{\nu}^{2}}
$$

where the coefficients $a_{i}$ depend on the parent nucleus. The values of $a_{i}$ for the different isotopes we used are tabulated in $[18,13]$. These expressions are very good approximations of the (measured) reactor flux for values of $E_{\nu} \geqslant 2 \mathrm{MeV}$.

The cross section for $\bar{\nu}_{e}+p \rightarrow e^{+}+n$ has been computed including corrections related to the recoil momentum of the daughter neutron in [19]. It should be noted that the energy spectrum of antineutrinos produced at nuclear reactors has been measured with good accuracy at previous reactor neutrino experiments (see [10] for references). For this reason, we will assume that the expected (unoscillated) antineutrino energy spectrum is known precisely. Some of the effects of uncertainties in the incoming flux on the determination of oscillation parameters have been studied in [13], and are supposedly small.

Finally, we have to include the effects of neutrino oscillations. Here, we will constrain ourselves to the simplest two-level system, i.e., we assume $\nu_{e} \leftrightarrow \nu_{\text {other }}$ oscillations governed by only one mass-squared difference $\Delta m^{2}$ (which is the "solar" $\Delta m^{2}$ ) and one mixing angle [we choose $\tan ^{2} \theta$ as the physical variable in order to separate the light side $(\theta$ $<\pi / 4)$ from the dark side $(\theta>\pi / 4)$ of the parameter space $[15,20]]$. The reason for this is that, according to [11], the KamLAND experiment should not have enough sensitivity to distinguish a nonzero $U_{e 3}$ from zero, given the current bound provided by the $\mathrm{CHOOZ}$ experiment [21]. This being the case, the (energy dependent) electron antineutrino survival probability at KamLAND is

$$
P\left(\bar{\nu}_{e} \leftrightarrow \bar{\nu}_{e}\right)=1-\sum_{i} f_{i} \sin ^{2} 2 \theta \sin ^{2}\left(\frac{1.27 \Delta m^{2} L_{i}}{E_{\nu}}\right),
$$

where $L_{i}$ is the distance of reactor $i$ to KamLAND in $k m, E_{\nu}$ is in $\mathrm{GeV}$ and $\Delta m^{2}$ is in $\mathrm{eV}^{2}$, while $f_{i}$ is the fraction of the total neutrino flux which comes from reactor $i$ (see [10]), which we assume is constant as a function of time. ${ }^{1}$

With all these ingredients at hand, we simulate KamLAND data for different values of $\tan ^{2} \theta$ and $\Delta m^{2}$ in 12 visible energy bins of width $500 \mathrm{keV}$, restricting ourselves to neutrino energies above $2 \mathrm{MeV}$ and below $8 \mathrm{MeV}$. The width of the bin is chosen according to the expected energy resolution, the low energy cutoff is included in order to respect the validity of Eq. (2.1), and the high energy cutoff is included in order to avoid bins with too few events, such that a Gaussian approximation for the statistical errors can be safely used. Finally, we define one "KamLAND-year" as the amount of time it takes KamLAND to see 800 events with visible energy above $1.22 \mathrm{MeV}$. This is roughly what is expected after one year of running (assuming a fiducial volume of 1 kton), if all reactors run at (constant) $78 \%$ of their maximal power output [10].

Our simulated data sets are analyzed via a standard $\chi^{2}$ function,

$$
\chi^{2}\left(\tan ^{2} \theta, \Delta m^{2}\right)=\sum_{j=1}^{N_{\text {bin }}} \frac{\left[N_{j}-T_{j}\left(\tan ^{2} \theta, \Delta m^{2}\right)\right]^{2}}{\left(\sqrt{N_{j}}\right)^{2}}+N_{\text {DOF }},
$$

where $N_{j}$ is the number of simulated events in the $j$-th energy bin, and $T_{j}\left(\tan ^{2} \theta, \Delta m^{2}\right)$ is the theoretical prediction for this number of events as a function of the oscillation parameters. $N_{\text {bin }}=12$ is the total number of bins, while $N_{\text {DOF }}$ is the number of degrees of freedom. This is done in order to estimate

\footnotetext{
${ }^{1}$ This need not be the case. Indeed, different reactors shut down at different times of the year, implying that $f_{i}$ does vary as a function of time. This effect, of course, will be considered in the analysis of real experimental data.

${ }^{2}$ This corresponds to a visible energy of roughly $1.22 \mathrm{MeV}$.
} 

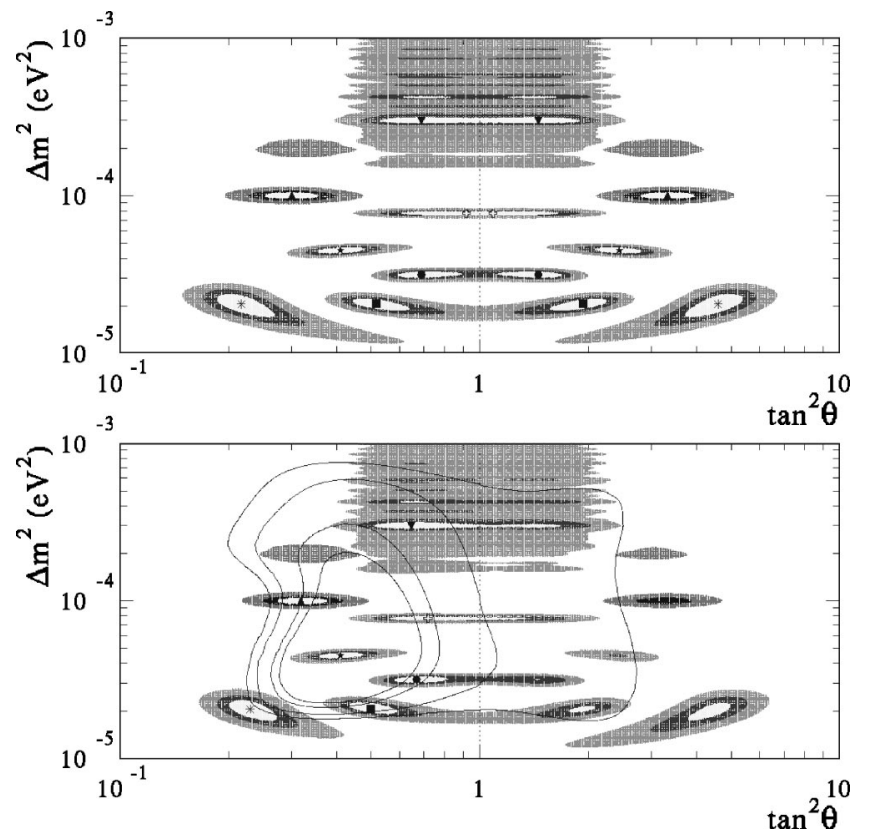

FIG. 1. Top: Region of the $\left(\Delta m^{2} \times \tan ^{2} \theta\right)$ parameter space allowed by three KamLAND-years of simulated data (see text) at the $90 \%$, three sigma and five sigma confidence levels (C.L.), for different input values of $\Delta m^{2}$ and $\tan ^{2} \theta$. The different symbols (star, square, circle, etc.) indicate the best fit points. Bottom: Same as top, except that the current solar data is also included in the fit, assuming that the electro-type neutrino oscillates into a pure active state. The line contours indicate the current LMA region, defined for 2 DOF, at $90 \%, 95 \%, 99 \%$, and three sigma C.L.

the statistical capabilities of an average experiment. ${ }^{3}$ An alternative option would be to include random statistical fluctuations in the simulated data. In this case, the appropriate definition of $\chi^{2}$ would be Eq. (2.3) minus $N_{\text {DOF }}$. Note that we assume statistical errors only, and do not include background induced events. This seems to be a reasonable assumption, given that KamLAND is capable of tagging the $\bar{\nu}_{e}$ by looking for a delayed $\gamma$ signal due to the absorption of the recoil neutron. However, "geophysical" $\bar{\nu}_{e}$ may prove to be irreducible background at the low energy bins $\left(E_{\text {visible }}\right.$ $\$ 2.6 \mathrm{MeV}$ ) [14]. For this reason, we discuss in the Appendix results of a modified analysis of KamLAND "data," where the events contained in the lowest energy bins are discarded.

\section{KamLAND SEES EVIDENCE FOR $\bar{\nu}$ DISAPPEARANCE}

If the solution to the solar neutrino puzzle lies in the LMA region, KamLAND should not only see a signal, but also determine $\tan ^{2} \theta, \Delta m^{2}$ with unprecedented precision [1114]. Figure 1(top) depicts $90 \%$, three sigma, and five sigma confidence level (C.L.) contours obtained for different simulated input values of $\tan ^{2} \theta, \Delta m^{2}$, after three KamLAND-

\footnotetext{
${ }^{3}$ See the Appendix A of [22] for a detailed discussion of this procedure.
}

years of "data." These contour are defined by plotting contours of constant $\Delta \chi^{2}\left(\tan ^{2} \theta, \Delta m^{2}\right) \equiv \chi^{2}\left(\tan ^{2} \theta, \Delta m^{2}\right)-\chi_{\min }^{2}$, while the C.L. are defined for two degrees of freedom $\left(\Delta \chi^{2}=4.61,11.83,28.76\right.$ for $90 \%$, three, and five sigma C.L., respectively).

Three important comments are in order. First, as observed in [12], for $\Delta m^{2} \geq 10^{-4} \mathrm{eV}^{2}$, the determination of $\Delta m^{2}$ is rather ambiguous. This is due to the fact that if $\Delta m^{2}$ is too large, the KamLAND energy resolution is not sufficiently high in order to resolve the oscillation lengths associated with these values of $\Delta m^{2}$ and $E_{\nu}$. Second, there is a discrete degeneracy in the allowed parameter space, as $\theta$ cannot be separated from $\pi / 2-\theta\left(\tan ^{2} \theta\right.$ from $\left.\tan ^{-2} \theta\right)$. Third, although $\tan ^{2} \theta, \Delta m^{2}$ can be very well determined, we have no information regarding whether the $\nu_{e}$ is oscillating into an active neutrino, a sterile neutrino, or a linear combination of the two. The reason for this, as has been emphasized before, is that KamLAND sees only charged current events, and that matter effects are completely negligible at KamLAND-like baselines.

Next, we add our simulated KamLAND data to the current solar data, including the recent result published by SNO [3]. The procedure for analysing the solar data is the same as the one adopted in [5], where we refer the readers for details. In particular, the analysis includes the event rates measured at Homestake and SNO ("charged current" data only), the average of the event rates measured at SAGE, GALLEX. and GNO, and the SuperKamiokande data, binned in energy for the day and night events. Furthermore, we include the theoretical errors on the neutrino fluxes according to the results of the 2000 Bahcall-Pinsonneault-Basu (BPB 2001) calculations [23]. We define a combined $\chi^{2}$ function in the straightforward way:

$$
\begin{aligned}
\chi_{\text {combined }}^{2}\left(\tan ^{2} \theta, \Delta m^{2}\right)= & \chi_{\odot}^{2}\left(\tan ^{2} \theta, \Delta m^{2}\right) \\
& +\chi_{\text {KamLAND }}^{2}\left(\tan ^{2} \theta, \Delta m^{2}\right) .
\end{aligned}
$$

Figure 1(bottom) depicts 90\%, three sigma, and five sigma C.L. contours obtained for different simulated input values for $\tan ^{2} \theta, \Delta m^{2}$, using the combined solar data plus three KamLAND-years of "data," assuming that the $\nu_{e}$ oscillates into a pure active state. As before, we define the confidence level contours by plotting contours of constant $\Delta \chi^{2}$, this time computed with Eq. (3.1). Figure 1(bottom) also depicts the LMA region of the parameter which is allowed by current data at the $90 \%, 95 \%, 99 \%$, and three sigma C.L. [5].

A few important features should be readily noted. First, the size of the $90 \%$ and three sigma contours in the light side are almost identical when one compares Fig. 1(top) (KamLAND only) and Fig. 1(bottom) (KamLAND plus solar), with the exception of the point located at a very large value of $\Delta m^{2}=3 \times 10^{-4} \mathrm{eV}^{2}$ [indicated in Fig. 1(top) by an upside-down triangle]. At this point, the number of separated "islands" allowed at the three sigma level decreases slightly. The five sigma contours, on the other hand, are slightly reduced when the solar data is included in the fit. This is particularly true when the input values of the oscillation param- 
eters are "far" from the current LMA best fit point (indicated by a star). Second, the "mirror symmetry" about the maximal mixing axis $\left(\tan ^{2} \theta=1\right)$ is broken. Indeed, in most cases, the addition of the solar data allows one to claim a "strong hint," at more than $90 \%$ C.L., that the $\nu_{e}$ is predominantly light. At five sigma, however, none of the points depicted in Fig. 1 allows for a "discovery" of this (fundamental) fact. One curious feature is that, for very small values of $\Delta m^{2}$, the five sigma C.L. contour may be smaller in the light side than in the dark side, while the best fit point is well defined to be in the light side. This behavior is most clear in the point marked with an asterix, and is due to the fact that the region below $\Delta m^{2} \lesssim 1 \times 10^{-5} \mathrm{eV}^{2}$ and $\tan ^{2} \theta \lesssim 1$ is strongly disfavored by the absence of a day-night effect at SuperKamiokande.

In Sec. III A, we analyze in more detail the ability of the current solar data to separate the light from the dark side of the parameter space, once KamLAND data becomes available (and if an oscillation signal is observed). In Sec. III B, we discuss what a combined solar and KamLAND data analysis will have to say about a sterile component in $\nu_{e} \leftrightarrow \nu_{\text {other }}$ oscillations.

\section{A. Separating the light from the dark side}

In order to address this issue, we compute the following "discrimination function"

$$
\begin{aligned}
& F\left(\tan ^{2} \theta, \Delta m^{2}\right) \\
& \quad \equiv\left|\chi_{\odot}^{2}\left(\tan ^{2} \theta, \Delta m^{2}\right)-\chi_{\odot}^{2}\left[\tan ^{2}(\pi / 2-\theta), \Delta m^{2}\right]\right|,
\end{aligned}
$$

which is defined for $0<\theta<\pi / 4$. Figure 2 depicts contours of constant $F$ in the $\left(\tan ^{2} \theta \times \Delta m^{2}\right)$ plane for $10^{-5} \mathrm{eV}^{2}<\Delta m^{2}$ $<10^{-3} \mathrm{eV}^{2}$ and $0.1<\tan ^{2} \theta<1 . F$ is best interpreted in the following way: if the best fit point obtained by analyzing the solar data (combined or not with KamLAND data) is $\left(\tan ^{2} \theta_{*}, \Delta m_{*}^{2}\right)$, than the mirror symmetric point $\left[\tan ^{2}(\pi / 2\right.$ $\left.\left.-\theta_{*}\right), \Delta m_{*}^{2}\right]$ is ruled out at the $x$ C.L., where the value of $x$ is determined by $\Delta \chi^{2}=F\left(\tan ^{2} \theta_{*}, \Delta m_{*}^{2}\right)$ for two degrees of freedom. For example, if any of the points which lie on the $F=5.99$ contour in Fig. 2 happens to be the best fit point, its symmetric point on the "other" side will be ruled out at the 95\% C.L.

Figure 2 also depicts the LMA 99\% C.L. region (defined for two DOF). For a sizable portion of it, $F$ is bigger than 5.99, meaning that we can expect to be able to separate the dark from the light side of the parameter space at the $95 \%$ C.L. for a significant part of the currently preferred region of the solar neutrino parameter space. This conclusion depends, of course, on whether the KamLAND data by itself can discriminate the obtained best fit point from maximal mixing $\left(\tan ^{2} \theta=1\right)$. Note that, according to the current solar data, a five sigma "discovery" that the electron-type neutrino is predominantly light (or heavy), is not allowed by the current solar neutrino data. The largest value of $F=11.5$ occurs very close to the best fit point indicated by the solar data. At this point, one can tell the dark from the light side at the $99.68 \%$ C.L.

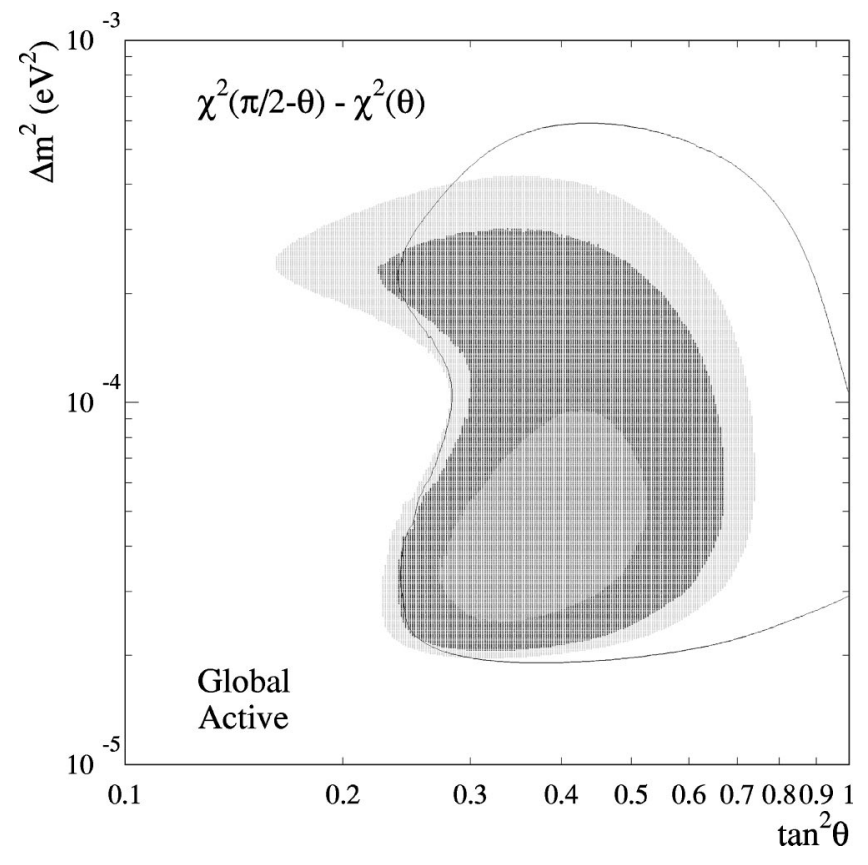

FIG. 2. Contours of constant $F \equiv\left|\chi_{\odot}^{2}(\theta)-\chi_{\odot}^{2}(\pi / 2-\theta)\right|$ in the $\left(\Delta m^{2} \times \tan ^{2} \theta\right)$ plane, for $10^{-5} \mathrm{eV}^{2}<\Delta m^{2}<10^{-3} \mathrm{eV}^{2}$ and 0.1 $<\tan ^{2} \theta<1$. The line contour corresponds to the LMA 99\% C.L. region, defined for $2 \mathrm{DOF}$ The regions correspond to $4.61<F$ $<5.99,5.99<F<9.21, F>9.21$ from the outermost to the innermost contour.

\section{B. Constraining $\nu_{e} \leftrightarrow \nu_{\text {sterile }}$ oscillations}

So far, we have assumed that $\nu_{e}$ oscillated into a pure active state $\nu_{a}$. Next we consider the more general case of $\nu_{e} \leftrightarrow \nu_{\text {other }}$ oscillations, where $\nu_{\text {other }}$ is a linear combination of active and sterile neutrinos. Explicitly, we define the mixing angle $\zeta$ such that

$$
\left|\nu_{\text {other }}\right\rangle=\cos \zeta\left|\nu_{a}\right\rangle+\sin \zeta\left|\nu_{s}\right\rangle .
$$

Note that we still assume that the $\nu_{e}$ is contained entirely in the two mass eigenstates whose mass difference is responsible for solving the solar neutrino puzzle. ${ }^{4}$ It is then possible to define oscillation probabilities, compute event rates at the various experiments in terms of $\Delta m^{2}, \tan ^{2} \theta$, and $\sin ^{2} \zeta$, and therefore compute $\chi_{\odot}^{2}\left(\tan ^{2} \theta, \Delta m^{2}, \sin ^{2} \zeta\right)$ [9]. Note that for KamLAND the situation is trivial, as $\chi_{\text {KamLAND }}^{2}$ is independent of $\sin ^{2} \zeta$.

In order to study what are the preferred values of $\sin ^{2} \zeta$, we follow the standard procedure of defining a one parameter $\chi^{2}$ function such that

$$
\chi^{2}\left(\sin ^{2} \zeta\right) \equiv \chi^{2}\left(\tan ^{2} \theta_{\min }, \Delta m_{\min }^{2}, \sin ^{2} \zeta\right),
$$

\footnotetext{
${ }^{4}$ In a four neutrino oscillation scheme, if we define $\nu_{1}$ and $\nu_{2}$ to be the mass eigenstates whose mass squared difference can be associated to $\Delta m_{\odot}^{2}$, this approximation corresponds to $\left|U_{e 1}\right|^{2}+\left|U_{e 2}\right|^{2}$ $=1$, while $\sin ^{2} \zeta \equiv\left|U_{s 1}\right|^{2}+\left|U_{s 2}\right|^{2}[9]$.
} 


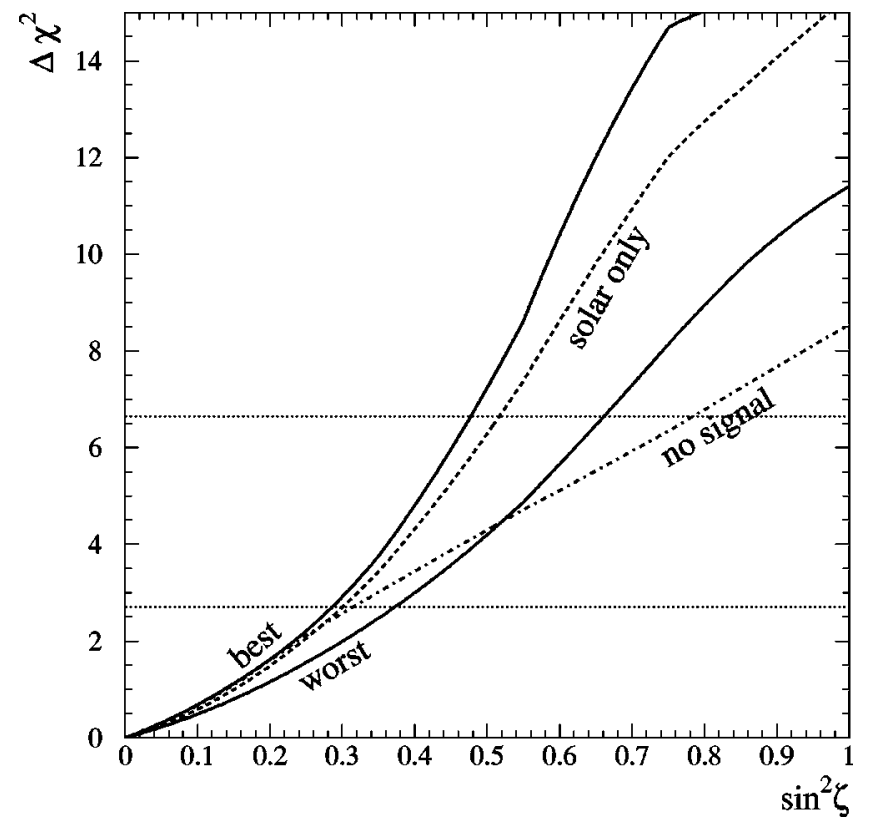

FIG. 3. $\Delta \chi^{2}\left(\sin ^{2} \zeta\right)$ as a function of $\sin ^{2} \zeta$, assuming that three KamLAND-years of "data" are consistent with two different points in the $\left(\Delta m^{2} \times \tan ^{2} \theta\right)$ plane (solid lines, "best" and "worst"), including only solar data in the fit (dashed line, "solar only"), or assuming that the three KamLAND-years of "data" do not contain any evidence for $\bar{\nu}_{e}$ oscillations (dot-dashed line, "no signal"). The horizontal dotted lines indicate the values of $\Delta \chi^{2}$ corresponding to $90 \%$ and $99 \%$ C.L. limits.

where $\tan ^{2} \theta_{\min }, \Delta m_{\min }^{2}$ are the values of $\tan ^{2} \theta, \Delta m^{2}$ which minimize $\chi^{2}$ for each value of $\sin ^{2} \zeta$.

Figure 3 depicts $\Delta \chi^{2}\left(\sin ^{2} \zeta\right) \equiv \chi^{2}\left(\sin ^{2} \zeta\right)-\chi^{2}\left(\sin ^{2} \zeta_{\text {min }}\right)$ as a function of $\sin ^{2} \zeta$, for different scenarios. The curve labeled "best" is obtained assuming that three KamLAND-years of "data" are consistent with the current best fit point $\left(\tan ^{2} \theta\right.$ $=0.41, \Delta m^{2}=4.5 \times 10^{-5} \mathrm{eV}^{2}$, indicated in Fig. 1 by a star) to the solar data assuming pure $\nu_{e} \leftrightarrow \nu_{a}$ oscillations, while the curve labeled "worst" is obtained assuming that three KamLAND-years of data are consistent with the best fit point in the would be LMA region assuming pure $\nu_{e} \leftrightarrow \nu_{s}$ $\left(\tan ^{2} \theta=0.69, \Delta m^{2}=3.2 \times 10^{-5} \mathrm{eV}^{2}\right.$, indicated in Fig. 1 by a circle). The dashed line labeled "solar only" is obtained if only the current solar data are included in the fit.

Three important features should be noted. First, $\sin ^{2} \zeta=0$ is always the best fit point. This is information contained in the current solar data by itself, of course. Second, the solar data by itself sets an upper bound $\sin ^{2} \zeta<0.30,0.52$ at the 90\%, 99\% C.L., respectively. Third, if three KamLANDyears of data are consistent with the "best" point, the upper bounds on $\sin ^{2} \zeta$ become slightly tighter $\left(\sin ^{2} \zeta<0.28,0.48\right.$ at the $90 \%$, 99\% C.L.), while if three KamLAND-years of data are consistent with the "worst" point the bounds actually become looser $\left(\sin ^{2} \zeta<0.37,0.66\right.$ at the $90 \%, 99 \%$ C.L.).

The reason for this behavior is the following: while KamLAND does not have the ability to discriminate active from sterile oscillations, it affects $\chi^{2}\left(\sin ^{2} \zeta\right)$ as defined in Eq. (3.4) by modifying the values of $\tan ^{2} \theta_{\min }$ and $\Delta m_{\min }^{2}$. Roughly speaking, the KamLAND data almost "fixes"
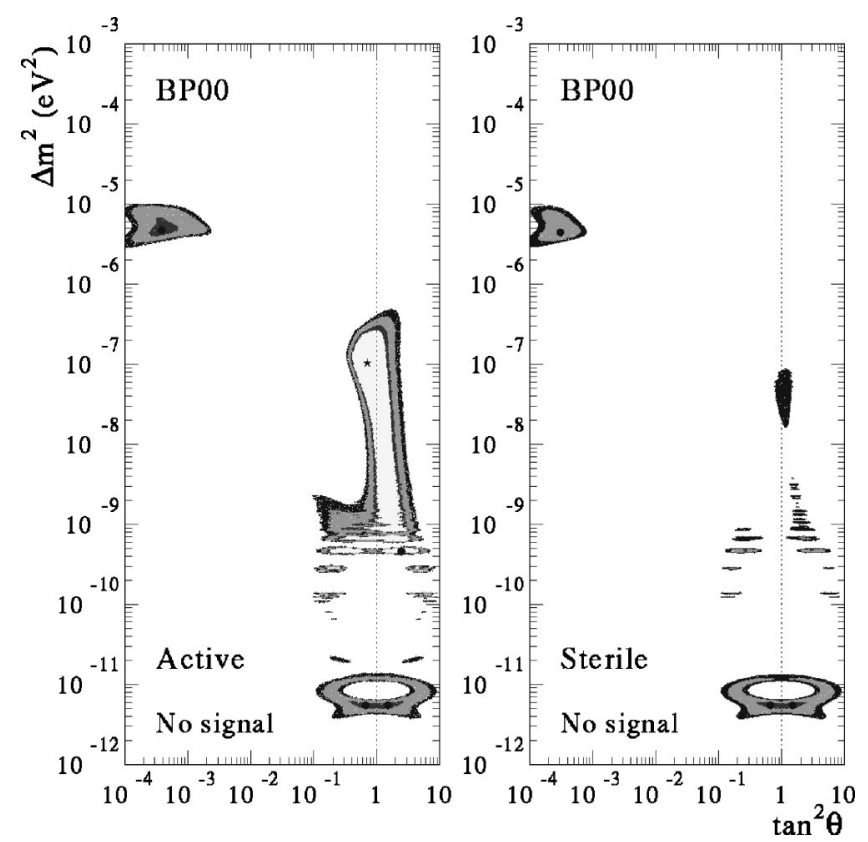

FIG. 4. Region of the $\left(\Delta m^{2} \times \tan ^{2} \theta\right)$ parameter space allowed by the current solar data plus three KamLAND-years of simulated data at the 90\%, 95\%, 99\%, and three sigma C.L. (for three degrees of freedom), assuming that KamLAND sees no evidence for neutrino oscillations. The left panel is for $\sin ^{2} \zeta=0$ (pure active oscillations), while the left panel corresponds to $\sin ^{2} \zeta=1$. The theoretical errors for the BP2000 neutrino fluxes are included in the analysis. See [5] for details on the treatment of the solar data.

$\tan ^{2} \theta_{\min }$ and $\Delta m_{\min }^{2}$ to the value chosen in the simulated KamLAND data [see Fig. 1(top)], independent of $\sin ^{2} \zeta$. At "best," this phenomenon is quite visible: it forbids one to change the value of $\tan ^{2} \theta$ and $\Delta m^{2}$ in order to obtain a better fit, and the upper bound on $\sin ^{2} \zeta$ becomes stronger. At "worst," a different manifestation of the same thing is present: the point "worst" is not particularly preferred by the current solar data, but the presence of KamLAND "forces" it to be the best fit point. It happens that the increase (with respect to the current best fit point) in $\chi_{\odot}^{2}$ is larger at $\sin ^{2} \zeta$ $=0$ than at $\sin ^{2} \zeta=1$, and for this reason the discrimination between pure active and pure sterile oscillations is poorer than the "solar only" case.

\section{KamLAND SEES NO EVIDENCE FOR $\bar{\nu}$ DISAPPEARANCE}

If the solution to the solar neutrino puzzle does not lie in the LMA region, the KamLAND data should be consistent with the hypothesis that the electron-type antineutrinos do not oscillate. Three KamLAND-years of "data" consistent with no oscillations prove enough to rule out the entire LMA region, at more than three sigma C.L. (see $[10,14]$ ).

Figure 4 depicts the "left-over" parameter space if this were the case. Note that, here, we are performing fits with three free parameters, $\left(\Delta m^{2}, \tan ^{2} \theta, \sin ^{2} \zeta\right)$, and Fig. 4 depicts the $\left(\tan ^{2} \theta \times \Delta m^{2}\right)$-plane for two fixed values of $\sin ^{2} \zeta=0,1$ (left and right, respectively). Confidence level contours are therefore computed for three degrees of freedom. 
As expected, Fig. 4 resembles Fig. 1 in [5], with the LMA solution "chopped off." Some quantitative differences are worthy of comment. For example, the best fit point is in the LOW region, while the entire range of values of $\Delta m^{2}$ which connect the LOW region to the VAC region (the QVO region) is allowed at more than $90 \%$ C.L. Furthermore, the SMA region, which is allowed by the current data only at the 99\% C.L. (according to the analysis performed in the spirit of [5]), would be allowed at the 95\% C.L. defined for three degrees of freedom.

If this happens to be the result obtained by the KamLAND experiment, we will probably have to wait for the upcoming Borexino experiment [24] in order to learn more about solar neutrino oscillations. In particular, the search for anomalous seasonal variations at Borexino should either exclude or establish the VAC and part of the QVO region, in which case oscillations parameters may be measured with reasonably good precision [22]. On the other hand, the search for a day-night asymmetry at Borexino should either exclude or establish the LOW and part of the QVO solution, in which case oscillations parameters may also be measured with reasonably good precision [25].

Finally, Fig. 3 depicts $\Delta \chi^{2}\left(\sin ^{2} \zeta\right)$ as a function of $\sin ^{2} \zeta$ if the KamLAND data does not see a signal for oscillations (dot-dashed line, labeled "no signal"). In this case, one would be able to set an upper bound $\sin ^{2} \zeta<0.32(0.78)$ at the 90\% (99\%) C.L., while at three sigma even values of $\sin ^{2} \zeta$ $=1$ would be allowed (as can be directly confirmed by looking at Fig. 4).

\section{SUMMARY AND CONCLUSIONS}

We have considered the consequences of combining the current solar neutrino data with future KamLAND reactor data. The main motivation for doing this is that while KamLAND can explore the entire LMA region of the solar neutrino solution space and measure the oscillation parameters with great precision, we will need extra input, which can only be provided by solar neutrino experiments (at least for the time being), in order to address two important issues: first, we would like to decide whether the electron-type neutrino is predominantly light or heavy, i.e., decide whether the solar angle is larger or smaller than $\pi / 4$ (in the convention where the mass-squared hierarchy of the mass eigenstates is fixed [15]). Second, we would like to know whether the electron-type neutrino mixes with the other known, active states (the muon- and tau-type neutrino), or whether it is (also) mixed with an unknown sterile neutrino state. It is worthwhile to recall that if the LSND anomaly [26] is also interpreted in terms of neutrino oscillations, sterile neutrinos exist.

We find that the current solar data do not allow a "discovery" (at the five sigma level) signal for $\theta<\pi / 4$ (or $>\pi / 4$ ) if KamLAND sees a signal for $\bar{\nu}_{e}$ disappearance, while a two sigma strong evidence may be claimed for a large part of the 99\% C.L. LMA region, if one assumes pure active oscillations. The situation is qualitatively similar if any "amount" of sterile mixing is present. ${ }^{5}$ The big caveat, of course, is that the KamLAND data should be able to rule out maximal mixing $\left(\tan ^{2} \theta=1\right)$ with comparable confidence.

We also find that while KamLAND has no power to discriminate active from sterile oscillations, the KamLAND data will modify the current upper bound on a sterile neutrino component in "solar" oscillations. Curiously enough, depending on the result obtained by KamLAND, this bound may improve or deteriorate when compared to an analysis performed with the current solar data only.

If KamLAND does not see evidence for $\bar{\nu}_{e}$ disappearance, the LMA region of the parameter space will be completely ruled out. The current solar data would, in this case, point to the LOW or QVO or VAC regions, while the SMA region would still be allowed at the two sigma level. If this is the case, data from the Borexino experiment will probably be required if we want to finally piece the solar neutrino puzzle.

We would like to emphasize that most of the results presented here and summarized in the previous paragraphs are obtained from the current solar data, and do not depend on the KamLAND "data." In particular, the conclusions we reach should apply whenever the KamLAND data is "precise enough" to either completely rule out the entire LMA region at a sufficiently high confidence level, or measure $\tan ^{2} \theta$ and $\Delta m^{2}$ values in the LMA region with significantly higher precision than the solar data. Until that is the case, the lack of convincing absence or presence of signal at KamLAND will either slowly "drown" the LMA solution (if it does not contain the correct solution to the solar neutrino puzzle) or create other potential degeneracies in the parameter space (one could picture a "best solar" versus "best KamLAND" degeneracy problem). Incidently, this type of "unresolved" scenario may indeed be upon us when the first results from KamLAND are released, perhaps before the Summer of 2002 [14].

Of course, as more solar data accumulates, the situation regarding the issues studied here may change. In particular, the SNO detector is still to measure the "neutral current" event rate [3], while more information will be obtained once the number of observed "charged current" events increases, and SNO is able to study the variation of the data sample as a function of time (day-night effect) and energy.

\section{ACKNOWLEDGMENTS}

We thank Concha Gonzalez-Garcia for many useful discussions and for collaboration during most of this work, and Alessandro Strumia for useful conversations. C.P.-G. also thanks the CERN Theory Division, where most of the work presented here was done, for its hospitality. This work was also supported by the Spanish DGICYT under grants PB980693 and PB97-1261, by the Generalitat Valenciana under

\footnotetext{
${ }^{5}$ Curiously, for small values of $\Delta m^{2} \sim 10^{-5} \mathrm{eV}^{2}$ and pure sterile oscillations, the data prefers values of $\theta$ in the dark side $\left(\tan ^{2} \theta\right.$ $>1$ ). Of course, such scenario is severely constrained by the current solar data (see Fig. 3).
} 

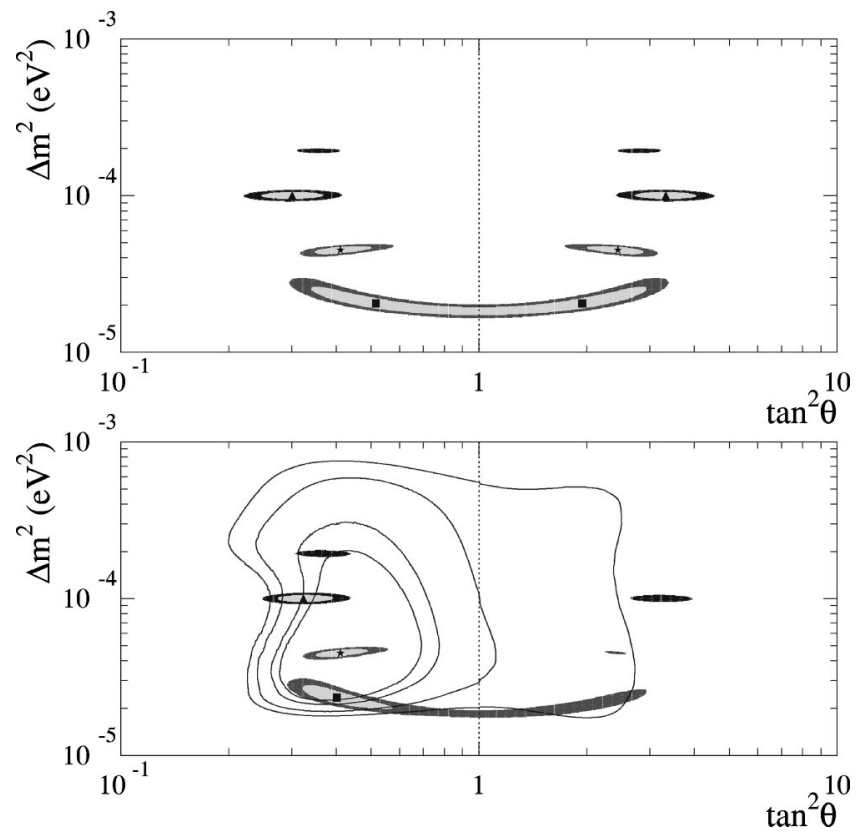

FIG. 5. Same as Fig. 1, when the three lowest energy bins at KamLAND are discarded from the "data" analysis. The confidence level contours correspond to $90 \%$ and three sigma (which correspond to $\Delta \chi^{2}=4.61$ and 11.83 , respectively).

grant GV99-3-1-01, and by the TMR network grant ERBFMRXCT960090 of the European Union and ESF network 86.

\section{APPENDIX: MORE CONSERVATIVE ANALYSIS OF KamLAND DATA}

The KamLAND data sample may be plagued by an irreducible background of low energy $\bar{\nu}_{e}$, produced in the surrounding rock [14]. The ultimate way of reducing this background is to introduce a visible energy threshold, above which the expected number of background events is negligible. According to [14], this cut should be placed at around 2.6 MeV.
In order to estimate the effect of this threshold, we repeat the analysis performed in Sec. III by only including the highest nine energy bins (corresponding to neutrino energies above $3.5 \mathrm{MeV}$, or visible energies slightly above $2.7 \mathrm{MeV}$ ). We again define one KamLAND-year as the time it takes KamLAND to observe, in the absence of oscillations, 800 events with visible energy above $1.22 \mathrm{MeV}$, such that the number of expected events above $2.7 \mathrm{MeV}$ is about 540 .

Figure 5(top) depicts $90 \%$ and three sigma C.L. contours obtained for different simulated input values for $\tan ^{2} \theta, \Delta m^{2}$, after three KamLAND-years of "data," excluding the three lowest energy bins. This is to be compared with Fig. 1(top) in Sec. III. Note that in Fig. 1 we display 90\%, three, and five sigma confidence level contours.

Figure 5(bottom) depicts $90 \%$ and three sigma C.L. contours obtained for different simulated input values for $\tan ^{2} \theta$, $\Delta m^{2}$, using the combined solar data plus three KamLANDyears of "data" minus the three lowest energy bins, assuming that the $\nu_{e}$ oscillates into a pure active state. This is to be compared with Fig. 1(bottom) in Sec. III.

Even if the C.L. contours are visibly bigger when one excludes the three lowest energy bins (as expected), three "KamLAND-years" is more than enough to obtain a clean "measurement" of $\Delta m^{2}$ and $\tan ^{2} \theta$, at least at the three sigma level. Of course, the problem of multiple solutions at large mass-squared differences now plagues even smaller values of $\Delta m^{2}$, while the precision with which one can determine oscillation parameters at $\Delta m^{2}$ close to $10^{-5} \mathrm{eV}^{2}$ is significantly worse, as expected. Note that, as we advertise in Sec. III A, the ability of the solar data to distinguish the light and dark sides does not depend on how well KamLAND can measure the oscillation parameters. Furthermore, the effect of the solar data in the analysis is more pronounced in the light side, especially at $\Delta m^{2}$ close to $10^{-5} \mathrm{eV}^{2}$. For example, while the KamLAND result is unable to exclude maximal mixing at $90 \%$ C.L. at the simulated point corresponding to $\Delta m^{2}=2 \times 10^{-5} \mathrm{eV}^{2}$ (labeled by a square), the inclusion of the solar data significantly reduces the $90 \%$.
[1] B.T. Cleveland et al., Astrophys. J. 496, 505 (1998).

[2] SuperKamiokande Collaboration, S. Fukuda et al., Phys. Rev. Lett. 81, 1158 (1998); 81, 4279(E) (1998); ibid. 82, 1810 (1999); 86, 5651 (2001); SuperKamiokande Collaboration, Y. Suzuki, Nucl. Phys. B (Proc. Suppl.) 91, 29 (2001).

[3] SNO Collaboration, Q.R. Ahmad et al., Phys. Rev. Lett. 87, 071301 (2001).

[4] G.L. Fogli et al., Phys. Rev. D 64, 093007 (2001).

[5] J.N. Bahcall, M.C. Gonzalez-Garcia, and C. Peña-Garay, J. High Energy Phys. 08, 014 (2001).

[6] A. Bandyopadhyay et al., hep-ph/0106264.

[7] P. Creminelli, G. Signorelli, and A. Strumia, J. High Energy Phys. 05, 052 (2001).

[8] V. Barger, D. Marfatia, and K. Whisnant, hep-ph/0106207.

[9] M.C. Gonzalez-Garcia, M. Maltoni, and C. Peña-Garay, Phys. Rev. D 64, 093001 (2001), and references therein.
[10] J. Busenitz et al., "Proposal for US Participation in KamLAND," 1999 (unpublished). May be downloaded from http:// bfk0.lbl.gov/kamland/

[11] V. Barger, D. Marfatia, and B.P. Wood, Phys. Lett. B 498, 53 (2001).

[12] R. Barbieri and A. Strumia, J. High Energy Phys. 12, 016 (2000).

[13] H. Murayama and A. Pierce, hep-ph/0012075.

[14] KamLAND Collaboration, K. Ishihara, talk at the NuFACT'01 Workshop in Tsukuba, Japan 2001. Transparencies at http:// psux1.kek.jp/ nufact01/index.html

[15] A. de Gouvêa, A. Friedland, and H. Murayama, Phys. Lett. B 490, 125 (2000).

[16] Kamiokande Collaboration, Y. Fukuda et al., Phys. Rev. Lett. 77, 1683 (1996); GALLEX Collaboration, W. Hampel et al., Phys. Lett. B 447, 127 (1999); SAGE Collaboration, J.N. Ab- 
durashitov et al., Phys. Rev. C 60, 055801 (1999); SAGE Collaboration, V.N. Gavrin, Nucl. Phys. B (Proc. Suppl.) 91, 36 (2001); GALLEX and GNO Collaborations, T.A. Kirsten, 77, 26 (1999); GNO Collaboration, M. Altmann et al., Phys. Lett. B 490, 16 (2000).

[17] Y. Declais et al., Phys. Lett. B 338, 383 (1995).

[18] P. Vogel and J. Engel, Phys. Rev. D 39, 3378 (1989).

[19] P. Vogel and J.F. Beacom, Phys. Rev. D 60, 053003 (1999).

[20] G.L. Fogli, E. Lisi, and D. Montanino, Phys. Rev. D 54, 2048 (1996).

[21] Chooz Collaboration, M. Apollonio et al., Phys. Lett. B 466, 415 (1999).
[22] A. de Gouvêa, A. Friedland, and H. Murayama, Phys. Rev. D 60, 093011 (1999).

[23] J.N. Bahcall, M.H. Pinsonneault, and S. Basu, Astrophys. J. 555, 990 (2001).

[24] Borexino Collaboration, G. Ranucci et al., Nucl. Phys. B (Proc. Suppl.) 91, 58 (2001).

[25] A. de Gouvêa, A. Friedland, and H. Murayama, J. High Energy Phys. 03, 009 (2001).

[26] LSND Collaboration, G.B. Mills, Nucl. Phys. B (Proc. Suppl.) 91, 198 (2001); LSND Collaboration, A. Aguilar et al., Phys. Rev. D (to be published), hep-ex/0104049. 